\title{
Solving Oscillating Problems Using Modifying Runge-Kutta Methods
}

Zainab Khaled Ghazal

Department of Mathematics, College of Science, Mustansiriyah University, Baghdad, Iraq.

zkgzainab@gmail.com

kasimabbas@uomustansiriyah.edu.iq

Article history: Received 11 April 2021, Accepted 30 May 2021, Published in October 2021.

Doi: $10.30526 / 34.4 .2703$

\begin{abstract}
This paper develop conventional Runge-Kutta methods of order four and order five to solve ordinary differential equations with oscillating solutions. The new modified RungeKutta methods (MRK) contain the invalidation of phase lag, phase lag's derivatives, and amplification error. Numerical tests from their outcomes show the robustness and competence of the new methods compared to the well-known Runge-Kutta methods in the scientific literature.
\end{abstract}

Keywords: Explicit Runge-Kutta methods, oscillating problems Phase-lag properties

\section{Introduction}

This essay dealing with the system of special first-order ordinary differential equation in the following form:

$$
u^{\prime}(t)=f(t, u), u\left(t_{0}\right)=u_{0}
$$

Such problems are often observed in various applied sciences, such as quantum chemistry, astronomy, quantum mechanics, electronics, elastics, and chemical physics (see [1,2]). Conventionally equation (1) is solved by using Runge-Kutta (RK) methods or two-step methods [3]. Several authors presented optimized numerical methods based on the phase lag characteristics (such as [4,5,6]). Exact and numerical solutions produce an angle between them is known as Phase-lag, and the distance of the numerical solution from the periodic solution is called amplification error. A five-stage four order Runge-Kutta method with phase-fitted and amplification-fitted proposed by Adel et al. [7]. Hussain et al. [8] proposed an optimized Runge-Kutta method to solve problem (1). The phase lag has the feature to develop more accurate numerical methods. This motivates us to propose the Runge-Kutta 
methods by using phase lag properties. Based on the previous work, we present a modified approach for the Runge-Kutta methods by combining the annulment of phase lag, phase lag's derivative, and amplification factor. The new MRK methods have been used to solve oscillation problems. The paper is orderly as follows: In Section 2, the phase-lag properties of the MRK method are given. In Section 3, we present how the new methods are derived. In section 4, we offer numerical results to illustrate the efficiency of the new MRK method. Section 5 is dedicated to Conclusions.

\section{Phase Lag Properties of MRK Methods}

We are concerned with the numerical solution of the ODEs of the form (1) with an oscillating solution. The general form of explicit MRK method for solving (1) is defined as follows:

$$
\begin{aligned}
& u_{n+1}=u_{n}+\sum_{i=1}^{s} b_{i} f\left(t_{n}+c_{i}, U_{i}\right) \\
& U_{i}=d_{i} u_{n}+h \sum_{j=1}^{i-1} a_{i j} f\left(t_{n}+c_{i} h, U_{j}\right), \quad i=1,2, \ldots, s .
\end{aligned}
$$

The corresponding Butcher tableau for equations (2) - (3) is expressed as follows

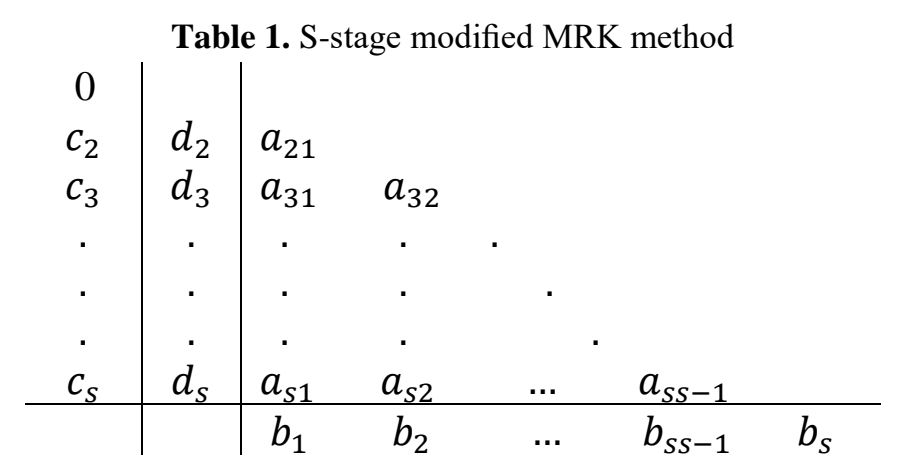

Constructing the new method depends on phase-lag and dissipation analysis proposed in [9]. To this goal, we use the following test equation,

$$
u^{\prime}=i w u, \quad w \in R
$$

An implementation of MRK method (2)-(3) to the test equation (4) we yield

$$
u_{n}=q_{*}^{n} u_{0}, \quad q_{*}^{n}=S\left(z^{2}\right)+i z V\left(z^{2}\right)
$$

where $z=w h$ and $S, V$ are polynomials in $z^{2}$ entirely specified by the coefficients $a_{i j}, c_{i}$ and $b_{i}$ of MRK method (2)-(3).

The comparison between equations (5) and (4) produces the following definition. Definition: [9] The following quantities in RK method (2)-(3)

$$
\begin{aligned}
& \text { 1- } P(z)=z-\arg \left[q_{*}(z)\right]=z-\tan ^{-1}\left(\frac{z \cdot V\left(z^{2}\right)}{S\left(z^{2}\right)}\right) \\
& \text { 2- } \left.D(z)=1-\left|q_{*}(z)\right|=1-\sqrt{\left(S^{2}\left(z^{2}\right)+z^{2} V^{2}\left(z^{2}\right)\right.}\right) .
\end{aligned}
$$

are respectively called phase lag or dispersion error and the amplification factor or dissipation error. If $P(z)=O\left(z^{r+1}\right)$ and $D(z)=O\left(z^{p+1}\right)$, then the method is said to be of dispersion order $r$ and dissipation order $p$.

\section{Derivation of new MRK methods}

This section proceeds to derive the modified RK method by abolishing phase lag, phase lag's derivative, and amplification error. 


\subsection{Five-Stage Fourth-Order Method}

A fourth-order MRK method of five-stage is given as follows [10]:

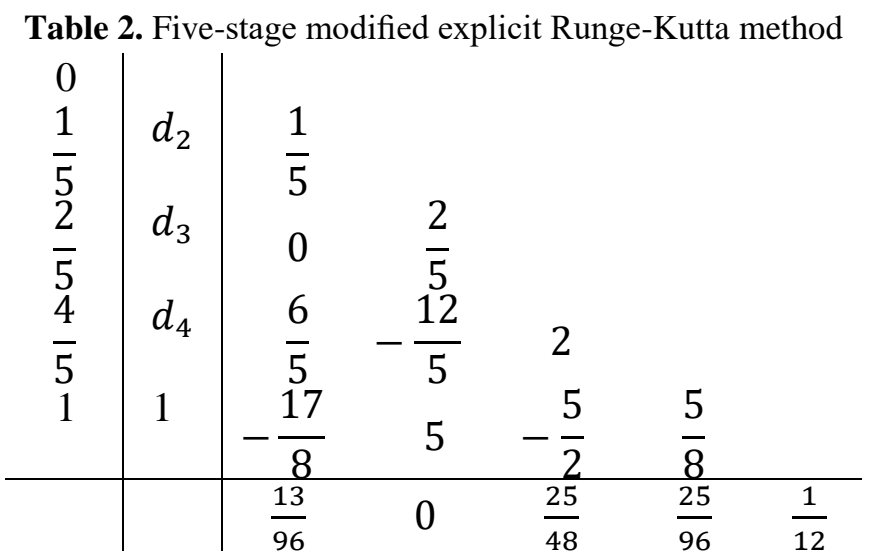

To construct a modified RK method, we set $d_{2}, d_{3}$, and $d_{4}$ as free parameters and the other parameters are the same as in Table 2. Motivated by the approach in [8], we obtain the phase lag, phase lag derivative and the amplification factor, which depend on $d_{2}, d_{3}$ and $d_{4}$ as follow:

$$
\begin{gathered}
P(z)=\tan (z)\left(\frac{1}{24} d_{2} z^{4}+\left(-\frac{5}{16} d_{3}-\frac{5}{96} d_{4}-\frac{13}{96}\right) z^{2}+1\right)-z\left(\frac{1}{120} z^{4}+\right. \\
\left.\left(-\frac{1}{16}-\frac{5}{48} d_{3}\right) z^{2}+\frac{7}{32}+\frac{25}{48} d_{3}+\frac{25}{96} d_{4}\right) \\
P^{\prime}(z)=\left(1+\tan ^{2}(z)\right)\left(\frac{1}{24} d_{2} z^{4}+\left(-\frac{5}{16} d_{3}-\frac{5}{96} d_{4}-\frac{13}{96}\right) z^{2}+1\right)+ \\
\tan (z)\left(\frac{1}{6} d_{2} z^{3}+2\left(-\frac{5}{16} d_{3}-\frac{5}{96} d_{4}-\frac{13}{96}\right) z\right)-\frac{1}{120} z^{4}- \\
\left(-\frac{1}{16}-\frac{5}{48} d_{3}\right) z^{2}-\frac{7}{32}-\frac{25}{48} d_{3}-\frac{25}{96} d_{4}-z\left(\frac{1}{30} z^{3}+2\left(-\frac{1}{16}-\frac{5}{48} d_{3}\right) z\right) \\
\frac{1}{14400} z^{10}+\left(-\frac{1}{960}+\frac{1}{576} d_{2}^{2}-\frac{1}{576} d_{3}\right) z^{8}+\left(\frac{5}{1152} d_{4}+\frac{29}{3840}+\frac{25}{1152} d_{3}+\right. \\
\left.\frac{25}{2304} d_{3}^{2}-\frac{5}{1152} d_{2} d_{4}-\frac{13}{1152} d_{2}-\frac{5}{192} d_{2} d_{3}\right) z^{6}+\left(-\frac{25}{1152} d_{3} d_{4}+\frac{25}{9216} d_{4}^{2}+\right. \\
\left.\frac{1}{12} d_{2}-\frac{25}{2304} d_{3}^{2}-\frac{83}{9216}-\frac{85}{4608} d_{4}-\frac{5}{192} d_{3}\right) z^{4}+\left(-\frac{685}{3072}-\frac{305}{768} d_{3}+\right. \\
\left.\frac{625}{9216} d_{4}^{2}+\frac{625}{2304} d_{3} d_{4}+\frac{625}{2304} d_{3}^{2}+\frac{5}{512} d_{4}\right) z^{2}
\end{gathered}
$$

Now, solving equations (6),(7), and (8), we get the values of free parameters in terms of $z$, and the expressions are too complicated, we use the following Taylor series expressions,

$$
d_{2}=1-\frac{29}{630} z^{2}-\frac{311}{75600} z^{4}-\frac{46369}{74844000} z^{6}-\frac{120976607}{1225944720000} z^{8}-
$$




$$
\begin{aligned}
& \frac{1756548533}{110335024800000} z^{10}-\frac{3475880890247}{1350500703552000000} z^{12}-\cdots, \quad d_{3}= \\
& 1-\frac{4}{1575} z^{4}-\frac{47}{94500} z^{6}-\frac{1009}{13365000} z^{8} \frac{1695769}{139311900000} z^{10}- \\
& 1-\frac{8}{1575} z^{4}-\frac{37}{47250} z^{6}-\frac{437}{11694375} z^{8} \frac{4558639}{766215450000} z^{10}- \\
& \frac{271269671}{137918781000000} z^{12}-\cdots, \quad d_{4}= \\
& \frac{32225033}{34479695250000} z^{12}-\cdots
\end{aligned}
$$

It can observe that for $z \rightarrow 0$, the new method reduces to an original RK method given in Dormand [10].

\subsection{Six-Stage Five-Order Method}

Consider the following six-stage modified RK method which can be expressed in Butcher tableau [3].

Table 3. Five-stage modified explicit Runge-Kutta method

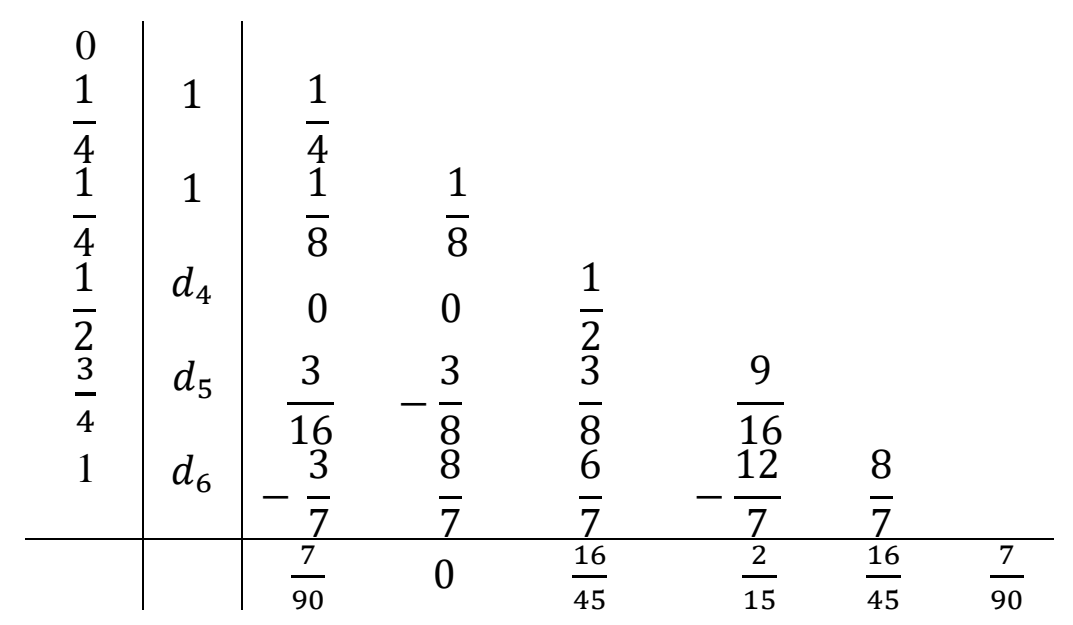

According to the method in Table 3, the phase lag, phase lag derivative, and amplification factor become

$$
\begin{aligned}
P(z)= & \tan (z)\left(1+\left(-\frac{31}{90}-\frac{1}{15} d_{4}-\frac{4}{45} d_{5}\right) z^{2}+\frac{1}{24} z^{4}-\frac{1}{1280} z^{6}\right)-z\left(\frac{7}{90} d_{6}+\right. \\
& \left.+\frac{13}{30}+\frac{2}{15} d_{4}+\frac{16}{45} d_{5}+\left(-\frac{7}{90}-\frac{1}{20} d_{4}\right) z^{2}+\frac{1}{120} z^{4}\right) \\
P^{\prime}(z)= & \left(1+\tan ^{2}(z)\right)\left(1+\left(-\frac{31}{90}-\frac{1}{15} d_{4}-\frac{4}{45} d_{5}\right) z^{2}+\frac{1}{24} z^{4}-\frac{1}{1280} z^{6}\right)+ \\
& \tan (z)\left(2\left(-\frac{31}{90}-\frac{1}{15} d_{4}-\frac{4}{45} d_{5}\right) z+\frac{1}{6} z^{3}-\frac{3}{640} z^{5}\right)-\frac{7}{90} d_{6}-\frac{13}{30}-\frac{2}{15} d_{4}- \\
& \frac{16}{45} d_{5}-\left(-\frac{7}{60}-\frac{1}{20} d_{4}\right) z^{2}-\frac{1}{120} z^{4}-z\left(\frac{1}{30} z^{3}+2\left(-\frac{7}{60}-\frac{1}{20} d_{4}\right) z\right),
\end{aligned}
$$


Ibn Al-Haitham Jour. for Pure \& Appl. Sci. 34(4)2021

$$
\begin{array}{r}
D(z)=\frac{1}{1638400} z^{12}+\frac{1}{230400} z^{10}+\left(\frac{19}{57600}-\frac{7}{9600} d_{4}+\frac{1}{7200} d_{5}\right) z^{8}+\left(\frac{1}{120} d_{4}-\right. \\
\left.\frac{1}{675} d_{5}+\frac{1}{400} d_{4}^{2}-\frac{163}{17280}+\frac{7}{5400} d_{6}\right) z^{6}+\left(-\frac{77}{2700} d_{4}-\frac{2}{225} d_{4}^{2}-\frac{44}{2025} d_{5}+\right. \\
\left.\frac{817}{8100}-\frac{49}{2700} d_{6}-\frac{7}{900} d_{4} d_{6}-\frac{16}{675} d_{4} d_{5}+\frac{16}{2025} d_{5}^{2}\right) z^{4}+\left(\frac{88}{675} d_{5}+\frac{4}{225} d_{4}^{2}+\right. \\
\frac{49}{8100} d_{6}^{2}+\frac{256}{2025} d_{5}^{2}+\frac{64}{675} d_{4} d_{5}+\frac{112}{2025} d_{5} d_{6}+\frac{14}{675} d_{4} d_{6}+ \\
\left.+\frac{91}{1350} d_{6}-\frac{4}{225} d_{4}-\frac{451}{900}\right) z^{2}
\end{array}
$$

Solving equations (10),(11), and (12), we find

$$
\begin{gathered}
d_{4}=1-\frac{25}{2016} z^{4}-\frac{31}{4536} z^{6}-\frac{613}{221760} z^{8}-\frac{116287}{103783680} z^{10}-\frac{5938297}{1307643680} z^{12}- \\
\frac{3273113521}{17784371404800} z^{14}-\cdots, \\
d_{5}=1+\frac{347}{21504} z^{4}+\frac{67}{13824} z^{6}+\frac{7367}{3548160} z^{8}+\frac{2819}{3354624} z^{10}+\frac{1827169}{5364817920} z^{12}+ \\
\frac{13092454033}{94849980825600} z^{14}+\cdots \\
d_{6}=1-\frac{247}{4704} z^{4}-\frac{1775}{84672} z^{6}-\frac{14143}{1552320} z^{8}-\frac{447763}{121080960} z^{10}-\frac{22865693}{15256200960} z^{12}- \\
\frac{2520658027}{4149686661120} z^{14}-\cdots
\end{gathered}
$$

It can demonstrate that for $z \rightarrow 0$, the new method same as the original RK method given in Butcher [3].

\section{Numerical Results}

To evaluate the performance of the new modified Runge-Kutta methods suggested in this paper, we apply them to five oscillatory problems and then compared the numerical results with the several well-known efficient methods. We use the criteria of absolute error to measure the accuracy of the method, which is given by

Absolute error $=\max \left(\left|y\left(t_{n}\right)-y_{n}\right|\right)$. Where $y\left(t_{n}\right)$ is the true solution and $y_{n}$ is the numerical solution. Figures 1-5 demonstrate the efficiency graphs of Log 10 (Max Error) versus step size $h$. Integration interval is [0, 1000] for all problems with step sizes $h=$ $0.1 / 2^{i}, i=1,2,3,4$. The following numerical methods are used in the comparison.

(i)

- MRK4: modified five-stage fourth-order RK method presented in Table 1 and Eqs. (9) in Section 3 in this paper.

- RK4D: the classical five-stage RK method of order four given in [10].

- RK4PF: the phase-fitted and amplification-fitted RK method given in [7].

- RK5S: six-stage RK method of order five given in [11].

- RK4MS: the modified four-order RK method given in [9]. 
- RK4B: the five-stage RK method of fourth-order given in [3].

(ii)

- MRK5: modified six-stage fifth-order RK method presented in Table 2 and Eqs. (13) in Section 3 in this paper.

- RK5B: the classical six-stage RK method of order five given in [3].

- ORK5T: Optimized fifth-order RK method given in [4].

- RK5M: Optimized RK method of order five given in [12].

- ORK5A: Optimized RK method of order five given in [5].

- RK5K: Optimized fifth-order RK method proposed in [6].

Problem 1: [13]

$$
u^{\prime \prime}(t)=100 u(t)+99 \sin (t), u(0)=1, \quad u^{\prime}(0)=11 .
$$

Whose exact solution is $u(t)=\cos (10 t)+\sin (10 t)+\sin (t)$, and $w=10$.

\section{Problem 2: [14]}

$$
u^{\prime \prime}(t)=-64 u(t), u(0)=1, \quad u^{\prime}(0)=-2 .
$$

Exact solution is $u(t)=\frac{1}{4} \sin (8 t)+\cos (8 t)$, and $w=8$.

\section{Problem 3: [15]}

$$
\begin{array}{ll}
u_{1}^{\prime \prime}(t)+u_{1}(t)=0.001 \cos (t), & u_{1}(0)=1, \quad u_{1}^{\prime}(0)=0, \\
u_{2}^{\prime \prime}(t)+u_{2}(t)=0.001 \sin (t), & u_{2}(0)=0, \quad u_{2}^{\prime}(0)=0.9995 .
\end{array}
$$

Exact solution is $u_{1}(t)=\cos (t)+0.0005 t \sin (t)$, $u_{2}(t)=\sin (t)-0.0005 t \cos (t)$, and $w=1$.

Problem 4: [16]

$$
\begin{gathered}
u^{\prime \prime}(t)+\left(\begin{array}{cc}
\frac{101}{2} & -\frac{99}{2} \\
-\frac{99}{2} & \frac{101}{2}
\end{array}\right) u(t)=\left(\begin{array}{c}
\frac{93}{2} \cos (2 t)-\frac{99}{2} \sin (2 t) \\
\frac{93}{2} \sin (2 t)-\frac{99}{2} \cos (2 t)
\end{array}\right), \\
u(0)=\left(\begin{array}{c}
0 \\
1
\end{array}\right), \quad u^{\prime}(0)=\left(\begin{array}{c}
-10 \\
12
\end{array}\right) .
\end{gathered}
$$

Exact solution and frequency are

$$
u(t)=\left(\begin{array}{c}
-\cos (10 t)(t)-\sin (10 t)+\cos (2 t) \\
\cos (10 t)+\sin (10 t)+\sin (2 t)
\end{array}\right), \quad w=10 .
$$

Problem 5: [17] Oscillatory system problem

$$
u^{\prime \prime}(t)+\left(\begin{array}{cc}
13 & -12 \\
-12 & 13
\end{array}\right) u(t)=\left(\begin{array}{c}
9 \cos (2 t)-12 \sin (2 t) \\
-12 \cos (2 t)+9 \sin (2 t)
\end{array}\right)
$$




$$
u(0)=\left(\begin{array}{l}
1 \\
0
\end{array}\right), \quad u^{\prime}(0)=\left(\begin{array}{c}
-4 \\
8
\end{array}\right)
$$

Exact solution and frequency are

$$
u(t)=\left(\begin{array}{c}
\sin (t)-\sin (5 t)+\cos (2 t) \\
\sin (t)+\sin (5 t)+\sin (2 t)
\end{array}\right), \quad w=5
$$

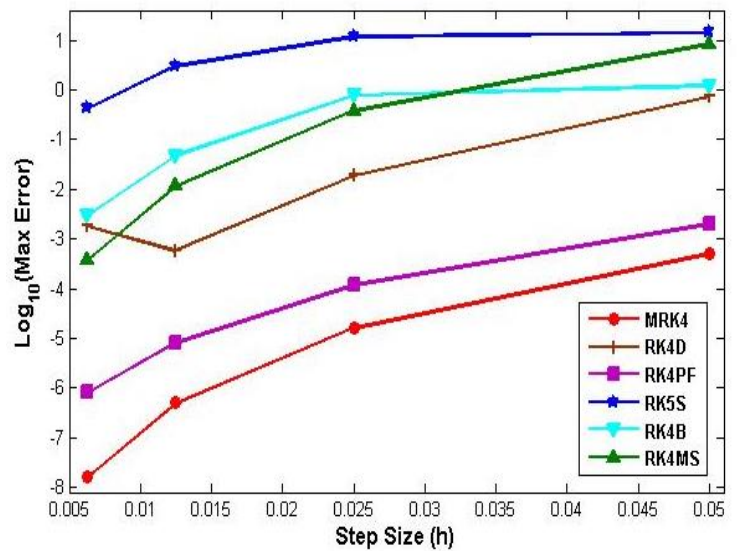

(a) Comparisons of the methods (i) in Section 4.

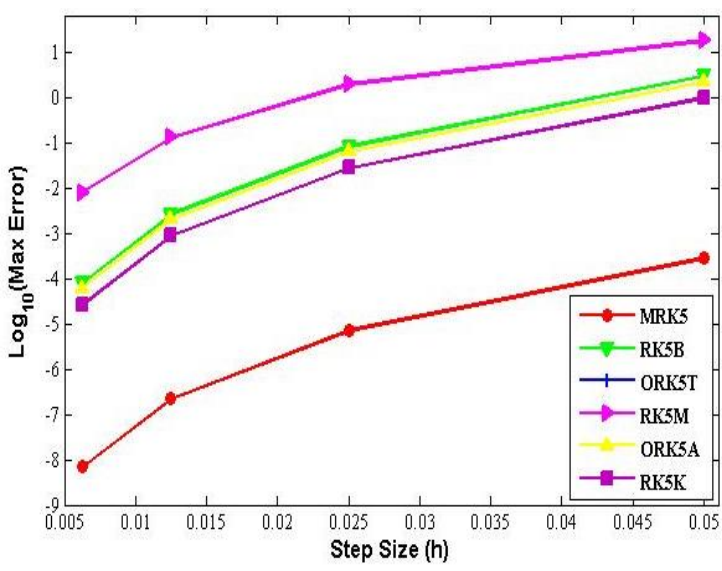

(b) Comparisons of the methods (ii) in Section 4.

Figure 1. The competence curves for Problem 1.

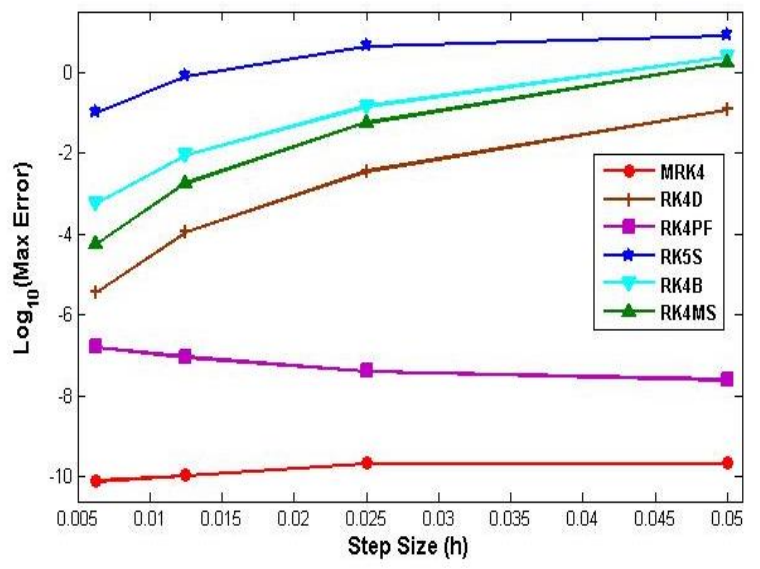

(a) Comparisons of the methods (i) in Section 4

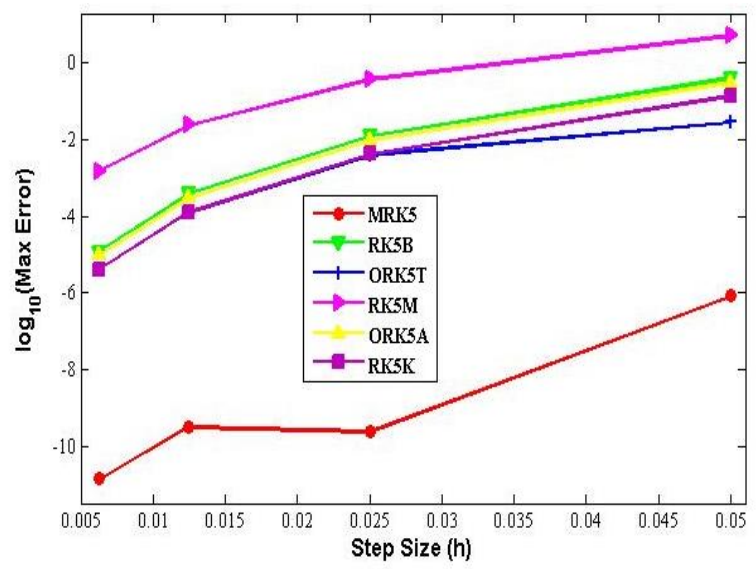

(b) Comparisons of the methods (ii) in Section 4.

Figure 2. The competence curves for Problem 2. 
Ibn Al-Haitham Jour. for Pure \& Appl. Sci. 34(4)2021

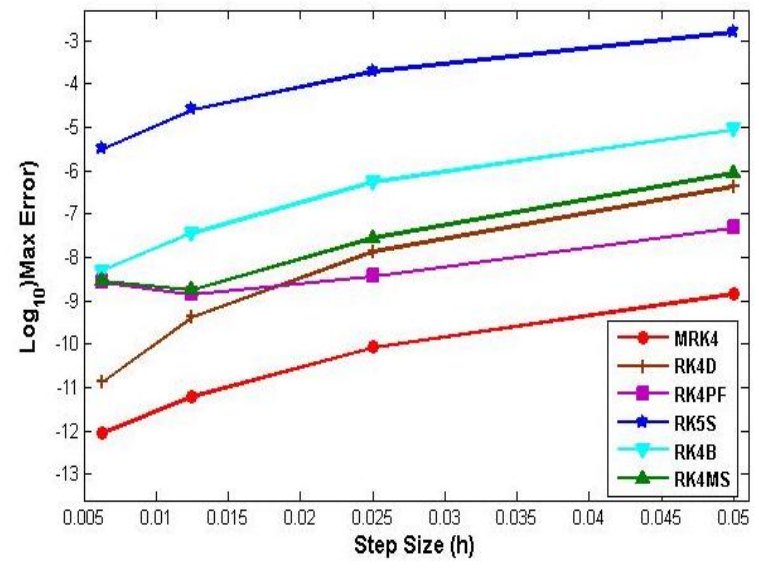

(a) Comparisons of the methods (i) in Section 4.

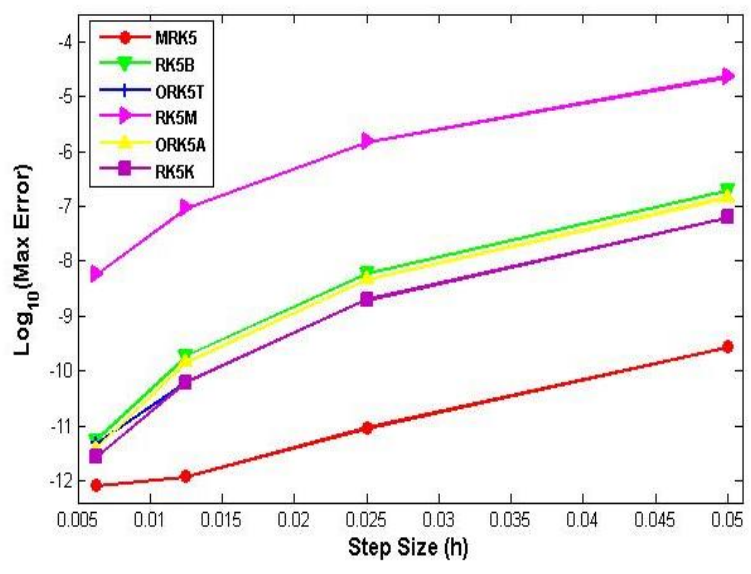

(b) Comparisons of the methods (ii) in Section 4.

Figure 3. The competence curves for Problem 3.

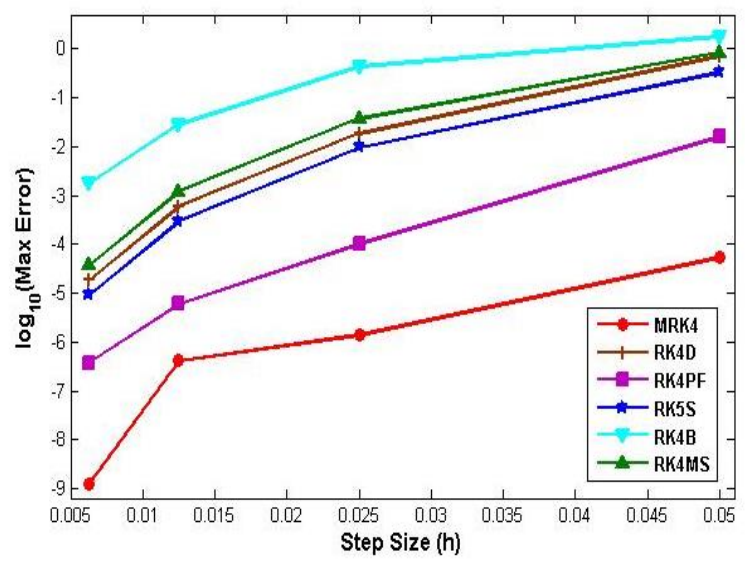

(a) Comparisons of the methods (i) in Section 4.

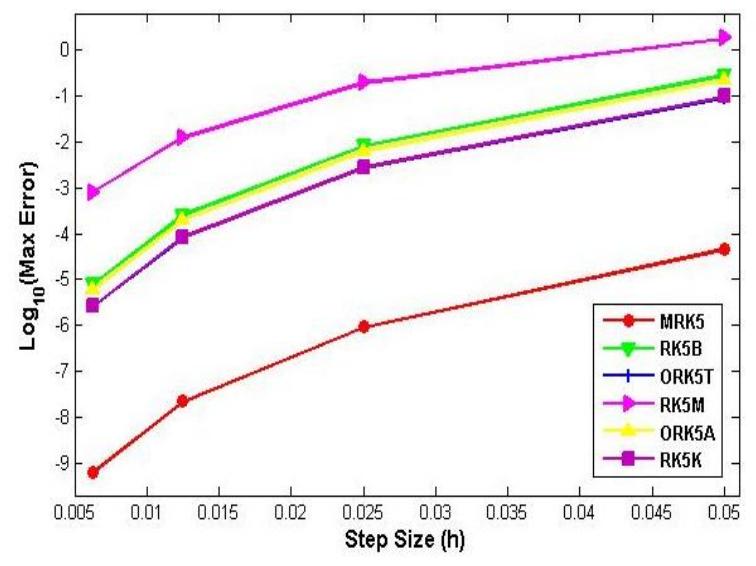

(b) Comparisons of the methods (ii) in Section 4.

Figure 4. The competence curves for Problem 4. 


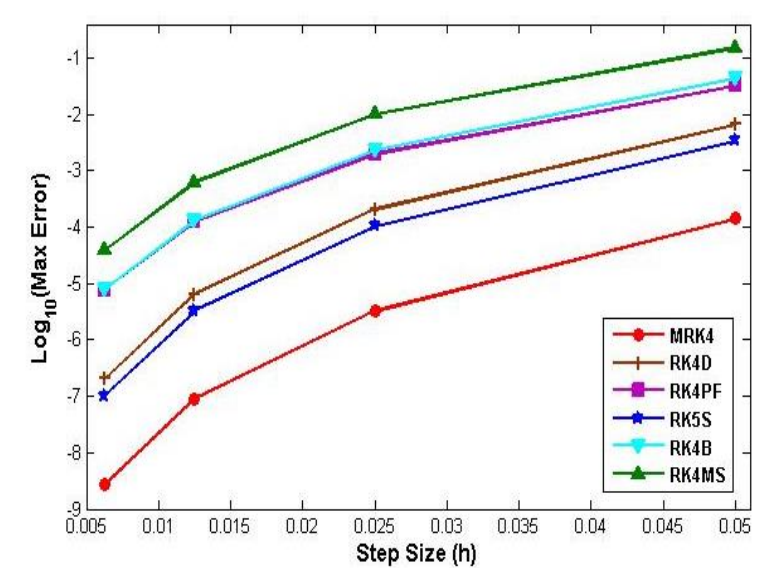

(a) Comparisons of the methods (i) in Section 4.

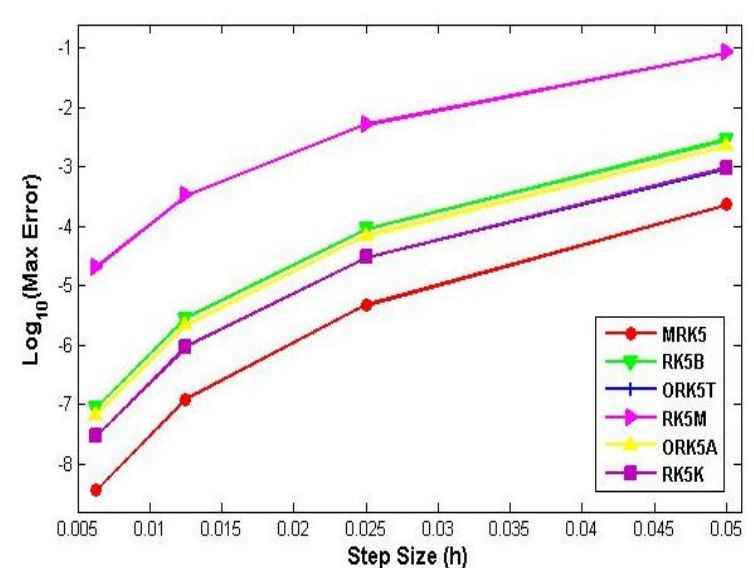

(b) Comparisons of the methods (ii) in Section 4.

Figure 5: The competence curves for Problem 5.

\section{Conclusion}

Classical Runge-Kutta methods are adjusted for solving the system of first-order ordinary differential equations whose solutions have a marked periodic style in this paper. The new methods are based on a fourth and fifth algebraic order given in [10] and [3], respectively. The proposed methods have nullified the phase-lag, amplification error, and phase lag's derivative. The results demonstrate the competence and effectiveness of the newly constructed methods, while they are compared to the standard methods and efficient RK methods. It is clear that the new MRK methods are the most accurate, and especially other than existing RK methods in the scientific literature.

\section{References}

1. Mahmood, B. S.; Gaftan, A. M.; Fawzi, F. A. Alternating Directions Implicit Method for Solving Homogeneous Heat Diffusion Equation. IHJPAS. 2020,33(2), 62-71, Doi: 10.30526/33.2.2427.

2. Al-Hawasy, J. A. A.; Mansour, N. F. The Galerkin-Implicit Methods for Solving Nonlinear Hyperbolic Boundary Value Problem. IHJPAS. 2021, 34(2), 119-128, Doi: 10.30526/34.2.2618.

3. Butcher, J. C. Numerical Methods for Ordinary Differential Equations, John Wiley \& Sons, 2nd edition, 2008, ISBN: 978-0-470-72335-7.

4. Tsitouras, C.; Simos, T. E. Optimized Runge-Kutta pairs for problems with oscillating solutions. Journal of Computational and Applied Mathematics, 2002, 147(2), 397-409. https://doi.org/10.1016/S0377-0427(02)00475-2.

5. Anastassi, Z. A.; Simos, T. E. Special optimized Runge-Kutta methods for IVPs with oscillating solutions. International Journal of Modern Physics C, 2004, 15(01), 1-15. https://doi.org/10.1142/S0129183104006510. 


\section{Ibn Al-Haitham Jour. for Pure \& Appl. Sci. 34(4)2021}

6. Kosti, A. A.; Anastassi, Z. A.; Simos, T. E. An optimized explicit Runge-Kutta method with increased phase-lag order for the numerical solution of the Schrödinger equation and related problems. Journal of mathematical chemistry, 2010, 47(1), 315-330. https://doi.org/10.1007/s10910-009-9571-z.

7. Fawzi, F. A.; Senu, N.; Ismail, F.; Majid, Z. A. New phase-fitted and amplification-fitted modified Runge-Kutta method for solving oscillatory problems. Global Journal of Pure and Applied Mathematics, 2016, 12(2), 1229-1242.

8. Hussain, K.; Ismail, F.; Senu, N.; Rabiei, F. Optimized fourth-order Runge-Kutta method for solving oscillatory problems. In AIP Conference Proceedings, 2016, 1739(1), 1-7. https://doi.org/10.1063/1.4952512.

9. Simos, T. E.; Aguiar, J. V. A modified Runge-Kutta method with phase-lag of order infinity for the numerical solution of the Schrödinger equation and related problems. Computers \& chemistry, 2001, 25(3), 275-281. https://doi.org/10.1016/S0097$\underline{8485(00) 00101-7 .}$

10. Dormand, J. R. Numerical methods for differential equations: a computational approach, CRC Press, New York. 1996, ISBN: 0-8493-9433-3.

11. Sakas, D. P.; Simos, T. E. A fifth algebraic order trigonometrically-fitted modified Runge-Kutta Zonneveld method for the numerical solution of orbital problems. Mathematical and computer modelling, 2005, 42(7-8), 903-920. https://doi.org/10.1016/j.mcm.2005.09.018.

12. Ming, Q.; Yang, Y.; Fang, Y. An optimized Runge-Kutta method for the numerical solution of the radial Schrödinger equation. Mathematical Problems in Engineering, 2012, 2012. https://doi.org/10.1155/2012/867948.

13. Salih, M.; Ismail, F.; Senu, N. Phase Fitted And Amplification Fitted Of Runge-KuttaFehlberg Method Of Order 4 (5) For Solving Oscillatory Problems. Baghdad Science Journal, 2020. 17(2), 689-693. https://dx.doi.org/10.21123/bsj.2020.17.2(SI).0689.

14. Ahmad, N. A.; Senu, N.; Ibrahim, Z. B.; Othman, M. Trigonometrically-Fitted Diagonally Implicit Two Derivative Runge-Kutta method for the Numerical Solution of Periodical IVPs. ASM Science Journal, 2019, 12(1), 50-59.

15. Demba, M. A.; Kumam, P.; Watthayu, W.; Phairatchatniyom, P. Embedded Exponentially-Fitted Explicit Runge-Kutta-Nyström Methods for Solving Periodic Problems. Computation. 2020, 8(32), 1-12. https://doi.org/10.3390/computation8020032.

16. Fawiz, F. A.; Gaftan, A. M.; Oguntunde, P. E.; Senu, N. An optimized Runge-Kutta method for the numerical solution of the oscillation problems. Journal of engineering and appliedsciences.2019, 14(19),17077-7083. https://doi.org/10.36478/jeasci.2019.7077.7083.

17. Hussain, K. A. Trigonometrically fitted fifth-order explicit two-derivative Runge-Kutta method with FSAL property. Journal of Physics: Conference Series. 2019, 1294(3), 1-10. https://doi.org/10.1088/1742-6596/1294/3/032009 